\title{
The Explanation of Magnetic Metal Carbon Mesocomposites Synthesis Peculiarities by Means of Mesoscopics Notions
}

\author{
Kodolov VI ${ }^{*}$, Kodolova-Chukhontzeva VV ${ }^{2}$, Terebova $\mathrm{NS}^{3}$ and Shabanova IN ${ }^{3}$ \\ ${ }^{1}$ Basic Research-High Educational Centre of Chemical Physics \& Mesoscopics, UD of RAS, Izhevsk, Russia \\ ${ }^{2}$ M.T. Kalashnikov Izhevsk State Technical University, Izhevsk, Russia \\ ${ }^{3}$ Udmurt Federal Research Centre, Ural Division, RAS, Izhevsk, Russia
}

Submission: November 11, 2019; Published: December 02, 2019

*Corresponding author: Kodolov VI, Basic Research - High Educational Centre of Chemical Physics \& Mesoscopics, UD of RAS, Izhevsk, Russia

\begin{abstract}
Mechanism of mesoparticles modification reactions are considered with the application of such notions as charges quantization, phase coherence, interference and annihilation. On the base of theoretical Mesoscopics ideas the formation of covalent bonds because of the interference of negative charges quants in modification reactions is discussed. The hypothesis about possibility of annihilation at the interaction of positive and negative charges quants in redox processes is presented. The magnetic metal carbon mesoscopic composites synthesis (for example, initial metal carbon Mesocomposites) is realized by mechanochemical method at the grinding of metal oxides microscopic particles with polyvinyl alcohol macromolecules. Then in the result the Copper or Nickel Carbon mesocomposites which have the following atomic magnetic moments: for Copper $-1,3 \mu \mathrm{B}$, for Nickel $-1,8 \mu \mathrm{B}$ are obtained. The investigations are carried out on the analysis examples of processes of Copper and Nickel Carbon mesoparticles modification by the compounds containing $\mathrm{p}, \mathrm{d}$ elements. In the middle of its such substances as polyethylene polyamine, ammonium iodide, ammonium polyphosphate (APP), silica $\left(\mathrm{SiO}_{2}\right)$, aluminum oxide, iron oxide, nickel oxide and copper oxide are used. It's noted that the red ox processes are accompanied by the metal atomic magnetic moments growth, that is explained by the electron shift on high energetic levels because of the annihilation phenomenon. The hypothesis concerning to the passing of two phenomena (annihilation and interference) at redox processes is proposed.
\end{abstract}

Keywords: Charges Quantization; Interference; Annihilation; Mesoparticles; Metal Carbon Mesocomposites; Red Ox Processes; Metal Atomic; Magnetic Moment

\section{Introduction}

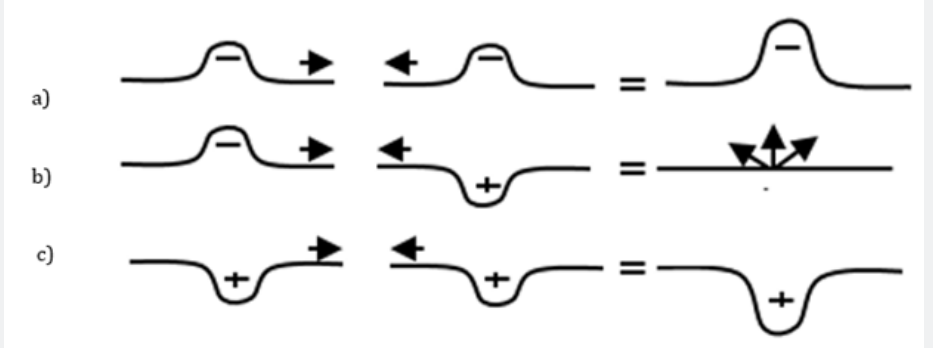

Figure 1: The scheme of charge quants interaction with appearance of interference (a), annihilation (b) and the formation of "dark hole" (c).

The process occurs at the charge's quantization with the certain phase coherence and then with the chemical bond's formation because of interference as well as in the red ox processes possible annihilation takes place [1,2]. If chemical reactions are realized without the changes of atoms oxidation states, then the negative charges quants quantization and the interference are carried out. However, the most reactions flow with the changes of elements oxidation states and then according to known schemes of reduction-oxidation processes it's necessary to take into consideration of positive charge quants. At the 
interaction of positive charge quants with the negative charge quants the annihilation phenomenon with the electromagnetic radiation or/and the direct electromagnetic field is possible. Also, the interaction of positive charges with the formation of "dark hole" must not excluded (Figure 1). In this case the explosion with diffusion of many most quantity of energy into surroundings is possible. The phenomena of charge quantization, interference and annihilation are considered on the examples of metal carbon mesoparticles interactions with reagents containing $\mathrm{p}$, d elements. At these investigations the basic method of researches is x-ray photoelectron spectroscopy.

\section{Results and Discussion}

The production of Metal Carbon mesoscopic composites is carried out with the using of mechanochemical interaction between microscopic particles of metal oxides and macromolecules of polymers at the active medium presence [3]. At the mesoscopic composites production, the sign variable loadings are applied. These loadings are appeared at the grinding with pressing. At the common grinding of Copper oxide particles with Polyvinyl alcohol (PVA) particles (or concentrated water solution) the metallic phase clusters falls between macromolecules of polyvinyl alcohol or, in other words, into reservoir (according to mesoscopic notions), in which banks are PVA macromolecules. The metal (example - Copper) within cluster has the positive charge. Therefore, the negative charge quants are directed to positive charged atom. In our case the negative charged quants from polyvinyl alcohol acetate and hydroxyl groups are transferred to copper positive charge quants. As a result, the annihilation with the electromagnetic direct field formation takes place. In this process the acetic acid and water are formed, and also the banks structures are changed: the poly acetylene and carbine fragments are appeared. There are unpaired electrons on joints of these fragments. The process of pair electron division and the shift of electrons on the high atomic levels for metal are explained by the annihilation origin. In this case the metal atomic magnetic moment growth is observed in the dependence on the electrons number which participates in red ox process.

The hypothesis about possibility of annihilation at the interaction of positive and negative charges quants in red ox processes is confirmed by the examples of processes of Copper and Nickel Carbon mesocomposites modification with application such substances as polyethylene polyamine, ammonium iodide, ammonium polyphosphate (APP), silica (SiO2), aluminum oxide, iron oxide, nickel oxide and copper oxide [3-5]. In the case, when polyethylene polyamine and ammonium iodide are applied, the connection reactions take place. At the interactions of polyethylene polyamine with mesoparticles the $\mathrm{C}=\mathrm{N}$ bond formation is explained by the interference of negative charges quants. When the mesoparticles modification reactions with the using APP, SiO2, metal oxides are carried out, the redox processes are realized. In these cases, the modifiers reduction reactions take place. The structures of metal carbon mesoscopic composites with active carbon shells are defined by means of the complex of methods including x-ray photoelectron spectroscopy, transition electron microscopy with high permission, electron microdiffraction and EPR spectroscopy. In correspondent reactions the element reduction for reagents and Nickel or Copper atomic magnetic moments growth in mesoparticles take place. Below in (Table 1) the examples of metal atomic magnetic moments changes for mesoparticles modified by APPh or silica after the mechanochemical modification processes are given. The presence of unpaired electrons on mesoparticles carbon shells in above systems is determined by means of electron paramagnetic resonance (EPR) (Table 2).

Table 1: The values of Copper (Nickel) atomic magnetic moments in the interaction products for systems:

\begin{tabular}{|c|c|c|c|}
\hline $\begin{array}{c}\text { Systems Cu C NC - substanc- } \\
\text { es }\end{array}$ & $\boldsymbol{\mu}_{\mathrm{cu}}$ & $\begin{array}{c}\text { Systems Ni C NC - } \\
\text { substance }\end{array}$ & $\boldsymbol{\mu}_{\mathbf{N i}}$ \\
\hline Cu C NC - silica & 3,0 & Ni C NC - silica & 4,0 \\
\hline Cu C NC - APPh & 2,0 & Ni C NC - APPh & 3,0 \\
\hline Cu C NC - APPh, relation 1:0,5 & 4,2 & & \\
\hline
\end{tabular}

$\mathrm{Cu} \mathrm{C} \mathrm{NC}-\mathrm{APPh}\left(\right.$ or $\mathrm{SiO}_{2}$ ) and $\mathrm{Ni} \mathrm{C} \mathrm{NC} \mathrm{-} \mathrm{APPh} \mathrm{(or} \mathrm{SiO}_{2}$ ).

Table 2: The unpaired electron values (from EPR spectra) for systems "Cu/C NC - silica" and "Cu/C NC - APPh" (relation 1:1) in comparison with mesoparticle $\mathrm{Cu} / \mathrm{C} \mathrm{NC}$.

\begin{tabular}{|c|c|}
\hline Substance & Quantity of unpaired electrons, spin/g \\
\hline $\mathrm{Cu} / \mathrm{C}$ nanocomposite & $1,2 \times 10^{17}$ \\
\hline system «Cu/C NC $-\mathrm{SiO}_{2} »$ & $3,4 \times 10^{19}$ \\
\hline system «Cu/C NC $-\mathrm{APPh} »$ & $2,8 \times 10^{18}$ \\
\hline
\end{tabular}

The metal atomic magnetic moment growth proceeds owing to the redox processes with above chemical compounds. In papers $[4,5]$ it's shown that the reduction reactions of Phosphorus and Silicon from correspondent substances at the interaction on the interphase boundary with mesoparticles are realized.

The relations of mesoparticles to above oxides are changed from 1:1 to 1:0,2 depending on the qualitive spectra obtaining, for example, the relations of 1:1 and 1:0,5 for system "Ni/C NC Al203" leads to full mask of mesoparticles. Therefore, the quantity of aluminum oxide is decreased to the relation 1:0,2. In accordance with Al3s spectra Aluminum is completely reduced during the modification process, and Nickel atomic magnetic moment is increased to $4,8 \mu \mathrm{B}$ (Table 3 ). In this case the reduction process is related to not only Aluminum oxide but also to Nickel oxide from metal cluster of mesoparticles (Ni C MC). Therefore, the reduction processes are stipulated by the electron transport from carbon shell of mesoparticles in direction to $\mathrm{Al}+3$ and $\mathrm{Ni}+2$ of atoms in correspondent oxides. Exceptional properties of modified Metal Carbon nanostructures with magnetic characteristics lead to the property's improvement of nanostructured polymeric coatings [6-8]. For example, the introduction of $0,008 \% \mathrm{Cu} \mathrm{C} \mathrm{MC} \mathrm{into}$ the melamine-formaldehyde resins stimulates the polarization growth in two times (on the AFM data). Similar results can be 
received at the combination of phase coherency and interference of charges quants during the preparation process of modified polymeric materials. The decreasing of nanostructures activity is possible when the modification is carried out with the ultrasound processing or the violation of phase coherency takes place at the nanostructures quantities changes $[9,10]$.

Table 3: Parameters of multiple splintering $\mathrm{Me} 3 \mathrm{~s}$ spectra in system $\mathrm{Ni} / \mathrm{C}+\mathrm{Al}_{2} \mathrm{O}_{3} . \mathrm{I}_{2} / \mathrm{I}$ - the relation of multiple splintering lines maximums intensities; $\Delta$ - Energetic distance between the multiple splintering maximums in Me3s spectra.

\begin{tabular}{|c|c|c|c|}
\hline Sample & $\mathbf{I}_{2} / \mathbf{I}_{\mathbf{1}} \mathbf{( N i )}$ & $\mathbf{\Delta}_{\text {Ni }} \mathbf{e V}$ & $\boldsymbol{\mu}_{\text {Ni }} \mu_{\text {5 }}$ \\
\hline $\mathrm{NiCMC}$ & 0.3 & 3 & 1.8 \\
\hline $\mathrm{NiC} \mathrm{MC}+\mathrm{Al}_{2} \mathrm{O}_{3}=1: 0.2$ & 0.9 & 1 & 4.8 \\
\hline
\end{tabular}

\section{Conclusion}

The present investigation has fundamental character. It's based on the ideas concerning to the change of Metal Carbon mesoscopic composites reactivity. The investigations are dedicated the mechanochemical red ox processes in which the electron transport from mesoscopic composite cluster to carbon shell takes place. In this case the electron delocalization is found. For the first time the metal carbon mesoscopic composite modification by mechanochemical process with the using of active substances including also bioactive systems is possible. The activity of metal carbon mesoscopic composites is caused by the structure and composition of correspondent composites, which contain the delocalized electrons and double bonds on the surface of carbon shell. Thus, at the mechanic chemical reduction/oxidation synthesis the changes of element oxidation states as well as the increasing of metal atomic moment for cluster can be appeared. At the same time, the modifiers elements and functional groups are discovered in carbon shell of mesoscopic composites modified. The creation of reactive mesoscopic materials with regulated magnetic characteristics which can find the application as modifiers of materials properties, catalysts for different processes, effective inhibitors of corrosion, sorbents, stimulators of plant growth, is very topical. These facts open new era for further investigations and development of metal carbon mesoscopic composites application fields.

\section{References}

1. Kodolov VI, Trineeva VV (2017) New scientific trend -Chemical Mesoscopics. Chemical Physics \& Mesoscopics 19(3): 454 - 465.

2. Yavorskiy BM, Detlaph AA (1965) Directory on Physics. Science pp. 848.

3. Kuhling H (1983) Reference Book on Physics. World pp. 519.

4. Rudenberg K (1964) Physical Nature of Chemical Bond. World pp. 162.

5. Kodolov VI, Trineeva VV, Kopylova AA et al. (2017) Mechanochemical modification of metal/carbon nanocomposites. Chemical Physics \& Mesoscopics 19(4): 569-580.

6. Shabanova IN, Kodolov VI, Terebova NS, Trineeva VV (2012) X ray electron spectroscopy in investigations ofmetal/carbon nanosystems and nanostructured materials. Udmurt University, Russia pp. 252.

7. Kodolov VI, Trineeva VV, Terebova NS (2018) Changes of electron structure and magnetic characteristics of modified copper/carbon nanocomposites. Chemical Physics \& Mesoscopics 20(1): 72-79.

8. Kopylova AA, Kodolov VV (2014) Investigation of copper/carbon nanocomposite interaction with silicium atoms from silicon compounds.

9. Chemical Physics \& Mesoscopics 16(4): 556 -560.

10. Wang JQ Wu WM, Feng DM (1992) The introduction to Electron Spectroscopy (XPS/XAES/UPS) -National Definite Industry Press, Beijing, China pp. 640.

\section{Your next submission with Juniper Publishers will reach you the below assets}

- Quality Editorial service

- Swift Peer Review

- Reprints availability

- E-prints Service

- Manuscript Podcast for convenient understanding

- Global attainment for your research

- Manuscript accessibility in different formats ( Pdf, E-pub, Full Text, Audio)

- Unceasing customer service

Track the below URL for one-step submission https://juniperpublishers.com/online-submission.php 